Journal of Applied Fluid Mechanics, Vol. 9, Special Issue 2, pp. 223-233, 2016. Selected papers from the XIIth Franco - Quebec Inter-University

Symposium on Thermal Systems -2015

Available online at www.jafmonline.net, ISSN 1735-3572, EISSN 1735-3645.

DOI: $10.36884 /$ jafm.9.SI2.25791

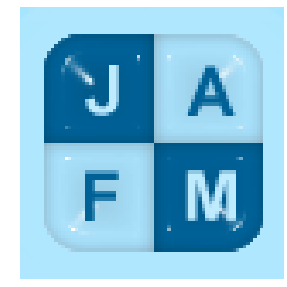

\title{
Numerical Approach of Thermal non Equilibrium Natural Convection in a Square Porous Cavity with Partially Thermally Active Side Walls
}

\author{
A. Omara ${ }^{1 \dagger}$, A. Bourouis ${ }^{1}$ and S. Abboudi ${ }^{2}$ \\ ${ }^{1}$ University of Mentouri Brothers, Constantine 25000, Algeria \\ ${ }^{2}$ IRTES-M3M Laboratory, UTBM, Belfort 90000, France \\ †Corresponding Author Email:abdeslam_omara@yahoo.fr
}

(Received October 8, 2015; accepted December 10, 2015)

\begin{abstract}
A numerical study was conducted to investigate thermal non equilibrium natural convection heat transfer in a square porous cavity with partial active vertical walls. The left vertical side wall is partially heated while the right side wall is partially cooled. The remaining portions of the vertical side walls as well as the top and bottom of the cavity are adiabatic. Depending on the location of hot part and cold part, respectively on the left and right side walls, different cases may be obtained. In this study we are limited to two cases: Upper-Lower and Lower-Upper active walls. The Brinkman Forchheimer extended Darcy model is used in the present study. Moreover, the two equations model is used to take into account separately local temperatures of the fluid and the solid. The resulting governing equations are solved by finite volume method and the standard SIMPLER algorithm. Numerical experiments have been carried out for a wide range of parameters, namely: Rayleigh number, Darcy number, inter-phase heat transfer coefficient and modified conductivity ratio. The obtained results reveal that the location of the hot and cold parts on the vertical side walls has a significant influence on the flow structure and the rate of heat transfer within the enclosure.
\end{abstract}

Keywords: Partially active walls; Non-Darcy Brinkman-Forchheimer model; Numerical approach; Local thermal non-equilibrium model.

\section{NOMENCLATURE}

$\begin{array}{ll}C_{F} & \text { coefficient of form drag } \\ D a & \text { Darcy number }=K / L^{2} \\ F & \text { Forchheimer number }=C_{F} L /|K|^{1 / 2} \\ K & \text { permeability } \\ L & \text { cavity length } \\ H & \text { inter-phase heat transfer coefficient }=h L^{2} / \varepsilon k_{f} \\ h & \text { volumetric inter-phase heat transfer coefficient } \\ \alpha_{\mathrm{s}} & \text { thermal diffusivity of solid }\end{array}$

\section{INTRODUCTION}

Different types of porous materials have been used in convective flowdue to wide range of applications in engineering such as geothermal energy, compact heat exchangers, buildings insulation, agriculture, cooling of electronics devices and solar receivers. Detailed reviews can be found in the books by Nield and Bejan (1999), Ingham and Pop (2002) and Vafai (2000), to name a few.

$$
\begin{aligned}
& \alpha_{\mathrm{f}} \quad \text { thermal diffusivity of fluid } \\
& \gamma \quad \text { thermal conductivity ratio }=\varepsilon k_{f} /(1-\varepsilon) k_{s} \\
& \theta_{\mathrm{s}} \quad \text { non-dimensional temperature of solid } \\
& \theta_{\mathrm{f}} \quad \text { non-dimensional temperature of fluid } \\
& \varepsilon \quad \text { porosity } \\
& \text { Subscripts } \\
& \text { solid } \\
& \text { f fluid }
\end{aligned}
$$

In most of these applications, the local temperature difference between the two phases is neglected. That is, there is no heat transfer from one phase to another. Such situation is called local thermal equilibrium (LTE). When the Rayleigh number is important or when we are in the presence of a faster heat transfer due to a significant flow velocity as in the case of storage of thermal energy derived from a solar energy conversion system, where a heated fluid flows from the solar collectors into a bed of 
rocks. In such situation, one can expect that the local thermal equilibrium becomes invalid. Thus, the porous medium is said to be in local thermal non-equilibrium (LTNE) state. Hence, a significant temperature difference between the fluid and solid phases is observed.

From a practical point of view the local nonequilibrium thermal theory plays an important role in porous media such as microprocessor computers using porous metal foams (Calmidi and Mahajan 2000), drying/freezing of foods (Zorlia andRubiolo2005), heating microwave (Dincov et al. 2004), etc.

The majority of the available studies have considered thermal equilibrium between solid porous matrix and saturated fluid whereas the analysis devoted to porous media as a nonequilibrium system are to our knowledge scarce. Saeid (2004) has analyzed mixed convection in a vertical porous layer by considering the nonequilibrium model. He found that for a mixed convection region, the total average Nusselt number is more for lower Rayleigh number than that for higher Rayleigh number. Baytas and Pop (2002) have investigated steady state flow and heat transfer characteristics in a square cavity filled with a porous medium assuming a non-equilibrium model. They found that the behavior of the flow characteristics is substantially modified, especially those of the local heat transfer coefficients. Vitro et al. (2009) have presented an experimental study to elucidate the several causes of local thermal nonequilibrium, even in steady or quasi steady heat transfer processes in saturated porous media, and to evaluate the influence of structural characteristics of porous media and the presence of surfactant in the saturating liquid phase. Wu et al. (2015) have conducted a numerical simulation to study natural convection in a rectangular cavity filled with a heatgenerating porous medium by using the two-energy equation model. The top and bottom walls of the enclosure are adiabatic and the left and right walls are partially heated and partially cooled by sinusoidal temperature profile. It is found that the phase deviation has significant influence on fluid flow and heat transfer in the porous cavity. Furthermore, they found that the increase of interphase heat transfer coefficient leads to faster reduction of dimensionless total heat transfer rate with the increase of periodicity parameter. Badruddinet al. (2007) numerically investigated heat transfer by convection, conduction and radiation in a saturated porous medium enclosed in a square cavity by using a non-equilibrium model. Effect of various parameters such as the inter-phase heat transfer coefficient, modified conductivity ratio, radiation and Rayleigh number on local and average Nusselt numbers, isotherms and streamlines is analyzed. Pipal and Bera (2013) numerically investigated the influence of local thermal nonequilibrium state between solid porous matrix and saturated fluid on natural convection in a square as well as slender enclosure. They found that, in both (LTE as well as LTNE states), maximum heat transfer takes place at a minimum value of aspect ratio $A_{0}$ of $A$. Furthermore, in LTE State $A_{0}$ is almost independent of LTE parameter and lies in between 1 and 1.5, however, the same under LTNE state is function of modified thermal conductivity, and the value of $A_{0}$ may be as large as 6 . Baytas (2003) performed a numerical study on natural convection of a heat-generating porous cavity with isothermally cooled walls using thermal nonequilibrium model, and the heat generation in the porous cavity takes place within the solid phase. Abdedou and Bouhadef (2015) have conducted a numerical study in the perspective to determine under what circumstances the local thermal equilibrium is valid in forced convection through a porous channel, and to compare between two most local thermal non-equilibrium criteria used in the literature. They showed that low values of the solidto-fluid thermal conductivity ratio, Reynolds and Prandtl numbers and high values of the porosity and Biot number are found to have favorable effects to satisfy the local thermal equilibrium between solid and fluid phases.

The aim of this work is to investigate the nonDarican natural convection heat transfer in a square porous cavity with partial active vertical walls using a thermal non-equilibrium model. To the best of our knowledge, natural convection through porous medium confined in a square cavity partially heated and partially cooled under the assumption of LTNE has not been reported so far.

\section{Physical Model}

A schematic diagram of the two dimensional square cavity of length L, under the present investigation, is shown in Fig. 1(a) and Fig. 1(b for the two studied cases: Top-Bottom and Bottom-Top locations of heating and cooling portions of the vertical side walls), respectively. A portion of the left wall is kept at temperature $\theta_{\mathrm{h}}$ and a portion of the right wall is at temperature $\theta_{\mathrm{c}}$ with $\theta_{\mathrm{h}}>\theta_{\mathrm{c}}$. The remaining parts of the left and right walls as well as the horizontal walls of the cavity are insulated. The porous medium is considered to be homogenous and isotropic. The fluid is assumed to be incompressible and the gravity acts in the downward direction. The velocity components are taken in the $x$ and $y$ directions, respectively. The porous medium is assumed to be in local thermal non-equilibrium state with the saturating fluid, and the generalized non-Darcy model reported in Bera et al. (2014) is adopted for modeling the fluid flow in the porous cavity. The thermal properties of the fluid are kept constant except the density in the buoyancy term in the momentum equation, which is satisfied by the state equation (Boussinesq approximation).

With these assumptions, the conservation equations for mass, momentum and energy for steady, two dimensional flow in an isotropic, homogenous porous medium can be written in non-dimensional form using the dimensionless variables: 
$(X, Y)=(x, y) / L, \quad(U, V)=L\left(\rho c_{p}\right)_{f}(u, v) / \varepsilon k_{f}$

$P=p L^{2} /\left(\rho_{f} \alpha_{f}^{2}\right), \gamma=\varepsilon k_{f} /(1-\varepsilon) k_{s}$

$H=h L^{2} /\left(\varepsilon k_{f}\right), \quad \theta_{f}=\left(T_{f}-T_{0}\right) /\left(T_{H^{-}} T_{C}\right)$

$T_{0}=\left(T_{H}+T_{C}\right) / 2$ and $\alpha_{f}=k_{f} /(\rho C p)_{f}$

as follows:

$\frac{\partial U}{\partial X}+\frac{\partial V}{\partial Y}=0$

$U \frac{\partial U}{\partial X}+V \frac{\partial U}{\partial Y}+\varepsilon^{2} F U\left(U^{2}+V^{2}\right)^{1 / 2}=$

$-\frac{\partial P}{\partial X}+\varepsilon P r \nabla^{2} U-\varepsilon \frac{P r}{D a} U$

$U \frac{\partial V}{\partial X}+V \frac{\partial V}{\partial Y}+\varepsilon^{2} F V\left(U^{2}+V^{2}\right)^{1 / 2}=$

$-\frac{\partial P}{\partial Y}+\varepsilon \operatorname{Pr} \nabla^{2} V-\varepsilon \frac{P r}{D a} V+\varepsilon R a P r \theta_{f}$

$U \frac{\partial \theta_{f}}{\partial X}+V \frac{\partial \theta_{f}}{\partial Y}=\nabla^{2} \theta_{f}+H\left(\theta_{s}-\theta_{f}\right)$

$0=\nabla^{2} \theta_{s}+\gamma H\left(\theta_{f}-\theta_{s}\right)$

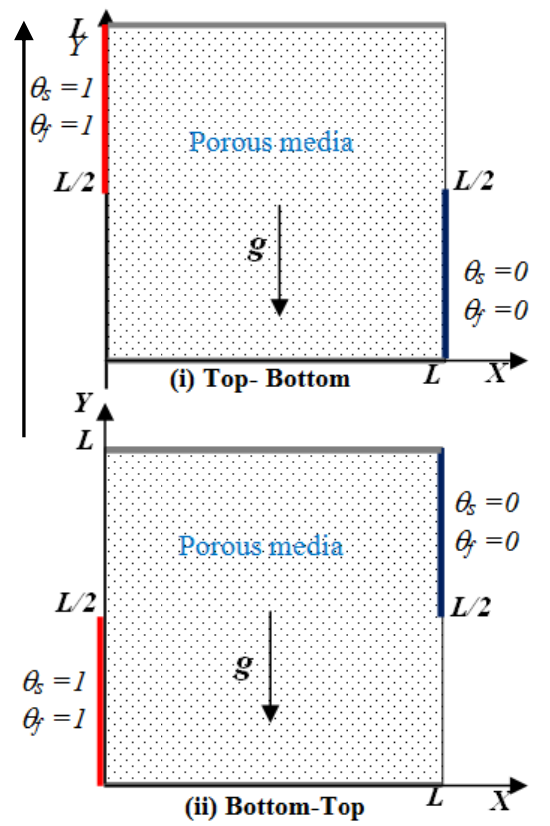

Fig. 1. Geometry and coordinate systems with different thermally active locations.

\section{NUMERICAL METHOD}

Governing Eqs. (1)-(5) with corresponding boundary conditions are discretized by the finite volume method (Patankar, 1980) using non-uniform grid. The diffusion term in the momentum and energy equations are approximated by the central difference scheme while the power law scheme is adopted for the convective terms. The SIMPLER algorithm is used to couple the velocity and pressure. The resulting set of discretized equations for each variable is solved by an iterative process.
The iterations are repeated until the convergence criterion: $\left(\varphi_{i, j}^{k+1}-\varphi_{i, j}^{k+1}\right) / \varphi_{i, j}^{k+1} \leq 10^{-5}$ is satisfied for each variable.

A grid independence study was carried out for various grid sizes as shown in Table lat $D a=10^{-3}, R a=10^{6}, \gamma=1$ and $H=10$. As one can see it in Table 1, no significant variation of the computational solutions is observed for the grid size greater than $80 \times 80$. Hence, the grid size of $80 \times 80$ is used for all the computations of the present study.

Table 1Grid independance test showing the effect of grid size on the average Nusselt numbers of fluid and solid

\begin{tabular}{|c|c|c|c|c|}
\hline $\begin{array}{c}\text { Control } \\
\text { volumes }\end{array}$ & $N u_{f}$ & $\begin{array}{c}\text { Change } \\
\%\end{array}$ & $N u_{s}$ & $\begin{array}{c}\text { Change } \\
\%\end{array}$ \\
\hline $20 \times 20$ & 4.553 & 3.07 & 2.154 & 3.11 \\
\hline $40 \times 40$ & 4.417 & 3.05 & 2.089 & 0.86 \\
\hline $60 \times 60$ & 4.286 & 1.51 & 2.071 & 0.33 \\
\hline $\mathbf{8 0} \times \mathbf{8 0}$ & $\mathbf{4 . 2 2 2}$ & $\mathbf{0 . 8 1}$ & $\mathbf{2 . 0 6 4}$ & $\mathbf{0 . 1 9}$ \\
\hline $100 \times 100$ & 4.188 & 0.48 & 2.060 & 0.19 \\
\hline $120 \times 120$ & 4.168 & - & 2.056 & - \\
\hline
\end{tabular}

Further, the accuracy of the numerical code developed by the authors is also validated with previously published numerical results obtained by Bera et al. (2014) in the case of double- diffusive natural convection in square porous medium with fully active vertical walls under thermal nonequilibrium state. The comparison is shown in Table 2. It can be observed from Table 2 that the results of the present simulation agree well with those reported by Bera et al. (2014).

The local and average Nusselt numbers for the solid and fluid phases along the partially vertical heated wall, are defined respectively as:

$$
\begin{aligned}
& N u_{S}=-\left(\frac{\partial \theta}{\partial X}\right)_{X=0}, \quad N u_{f}=-\left(\frac{\partial \theta}{\partial X}\right)_{X=0} \\
& \overline{N u}_{S}=\int_{Y_{a}}^{Y b}\left(\frac{\partial \theta s}{\partial X}\right)_{X=0} d Y, \overline{N u}_{f}=\int_{Y_{a}}^{Y b}\left(\frac{\partial \theta_{f}}{\partial X}\right)_{X=0} d Y
\end{aligned}
$$

Where:

$\left(Y_{a}, Y_{b}\right)=(0.5,1)$ for Top - Bottom location

$\left(Y_{a}, Y_{b}\right)=(0,0.5)$ for Bottom - Top location

\section{ReSUlts AND Discussion}

In this section, we try to clarify the effects of partially active thermal wall locations and local thermal non-equilibrium (LTNE) state between the porous matrix and the saturating fluid on naturalconvection in a cavity, and to analyze the effects of Rayleigh and Darcy numbers, thermal conductivity ratio and inter-phase heat transfer coefficient on the flow and heat transfer 
characteristics. The values of the above dimensionless parameters are varied in the range of $10^{3} \leq R a \leq 10^{6}, 0.1 \leq \gamma \leq 100,10^{-1} \leq D a \leq 10^{-6}$ and $0 \leq H \leq 1000$, while the Prandtl number $(\operatorname{Pr})$, the Forchheimer number $(F)$ and the porosity $(\varepsilon)$ are kept constant at $0.7,1$ and 0.9 , respectively.

In order to consider the effect of non-dimensional inter-phase heat transfer coefficient $(H)$, Fig. 2 shows the streamlines and isotherms for solid (on the right) and fluid (in the middle) for the case with Bottom-Top heating and cooling. The other parameters are all kept constant as $D a=10^{-3}$, $R a=10^{6}, \gamma=1$. As can be seen from the above figure that only the clockwise rotating convective cells appear in the streamlines plot whereas the variation of maximum magnitude of streamlines as a function of $H$ is significant. For the fluid contours, no significant change is observed. As $H$ is increased from 1 to 1000 modes of heat transfer in the solid changes from conduction to conduction as well as convection. Consequently, a significant change in the angle between solid temperature contour and sidewall is observed. At $H=1000$, the equilibrium between two phases is globally verified in view of the wide resemblance between the solid and liquid contours. It should be noted that the increase in $H$ is associated with an increase in the rate of heat transfer from the fluid phase to the solid phase. As a result, the local Nusselt number for the fluid is decreased with the increase of $H$ while the corresponding one for the solid is increased as can be observed from Fig. 3. Besides that, $N u_{f}$ reveals an increasing trend at the beginning of the heated zone and then undergoes a monotonic decrease until the end of the heated zone. The corresponding one for the solid phase shows a quasi-uniform variation at smaller values of $H$ on a large part of the heated zone. An increase of $N u_{s}$ is observed as $H$ is increased, while it decreases along the heated zone ( $Y$ direction) regardless of the $H$ values.

In order to investigate the effect of the location of the heating and cooling zones, we present in Fig. 4 the effect of $H$ on streamlines and isotherms for the case with Top-Bottom heating and cooling. A careful examination of this figure reveals that the location of heating-cooling has a major influence on the streamlines. The trend to form a two flow cells is noticed for this case for all values of $H$. The center of each cell shifted toward the adjacent thermally active portion. However, the intensity of the recirculation cell for this configuration remains less than that relating to the case with Bottom-Top heating and cooling. On the other hand, the temperature distribution for the solid and fluid phases is modified to be parallel to the horizontal walls at the center of the cavity at large values of $H$.

Table 2 Comparison of average Nusselt numbers (solid and fluid) and of average Sherwood number for a square porous cavity with $P r=0.7, \gamma=10$ and $F=0$

\begin{tabular}{|c|c|c|c|c|c|c|c|c|}
\hline \multirow{2}{*}{$H$} & \multirow{2}{*}{$D a$} & \multirow{2}{*}{$R a$} & \multicolumn{2}{|c|}{$N \boldsymbol{u}_{\boldsymbol{f}}$} & \multicolumn{2}{|c|}{$N \boldsymbol{u}_{\boldsymbol{s}}$} & \multicolumn{2}{c|}{$S \boldsymbol{c}$} \\
\cline { 3 - 9 } & & & Present & Beraet al.(2014) & Present & Beraet al.(2014) & Present & Beraet al.(2014) \\
\hline \multirow{3}{*}{0.1} & $10^{-2}$ & $10^{4}$ & 2.4897 & 2.4916 & 1.0320 & 1.0321 & 1.0380 & 1.0393 \\
\cline { 2 - 9 } & $10^{-4}$ & $10^{6}$ & 4.7366 & 4.8110 & 1.0470 & 1.0472 & 1.1351 & 1.1359 \\
\hline \multirow{3}{*}{1} & $10^{-2}$ & $10^{4}$ & 2.4680 & 2.4698 & 1.2608 & 1.2617 & 1.0387 & 1.0397 \\
\cline { 2 - 9 } & $10^{-4}$ & $10^{6}$ & 4.7039 & 4.7774 & 1.3926 & 1.3948 & 1.1361 & 1.1369 \\
\hline \multirow{3}{*}{10} & $10^{-2}$ & $10^{4}$ & 2.4300 & 2.4195 & 1.9507 & 1.9514 & 1.0411 & 1.0406 \\
\cline { 2 - 8 } & $10^{-4}$ & $10^{6}$ & 4.6166 & 4.6882 & 2.6266 & 2.6429 & 1.1387 & 1.1393 \\
\hline
\end{tabular}

Fig. 5 shows the fluid $\left(N u_{f}\right)$ and solid $\left(N u_{s}\right)$ local Nusselt numbers for $D a=10^{-3}, R a=10^{6}$ and $\gamma=1$. It is obvious that the local Nusselt numbers decrease from the beginning to the end of the heating zone contrary to the case with Bottom-Top heating and cooling where an increasing trend in the beginning of the heated zone is observed. At the end of the heated portion, both Nusselt numbers tend to values close to zero. In addition, it can observed that the fluid local Nusselt number $\left(N u_{f}\right)$ is practically independent of the inter-phase heat transfer coefficient, while the corresponding one of the solid $\left(N u_{s}\right)$ shows a certain degree of dependence. It is to be noted that for $H=1, N u_{s}$ is equal to unity over a large part of the active zone indicating that the heat transfer is purely due to conduction.

The effect of the location of partial thermally active vertical walls and inter-phase heat transfer coefficient on the dimensionless solid-to-fluid temperature difference at the horizontal mid-plane
$(Y=0.5)$ and at the vertical mid-plane $(X=0.5)$ of the cavity is presented in Fig. 6. By way of comparison, we present the case of total heating. It is clear that the inter-phase heat transfer coefficient $(H)$ has significant influence on the solid-to-fluid temperature difference. As $H$ increases, the solid-tofluid temperature difference decreases and approaches zero for large values, indicating that the LTE state is verified anywhere in the cavity. The maximum and minimum values of the solid-to-fluid temperature difference emerge close to the vertical active walls of the cavity. Whereas in the middle of the cavity a small effect is observed for all values of $H$ where the solid-to-fluid temperature difference gets close to zero except for the case with total heating at the vertical mid-plane $(X=0.5)$. At this position, the solid-to-fluid temperature difference decreases gradually from $Y=0$ to $Y=1$ at the point where the solid-to-fluid temperature difference is almost zero for all values of $H$. 

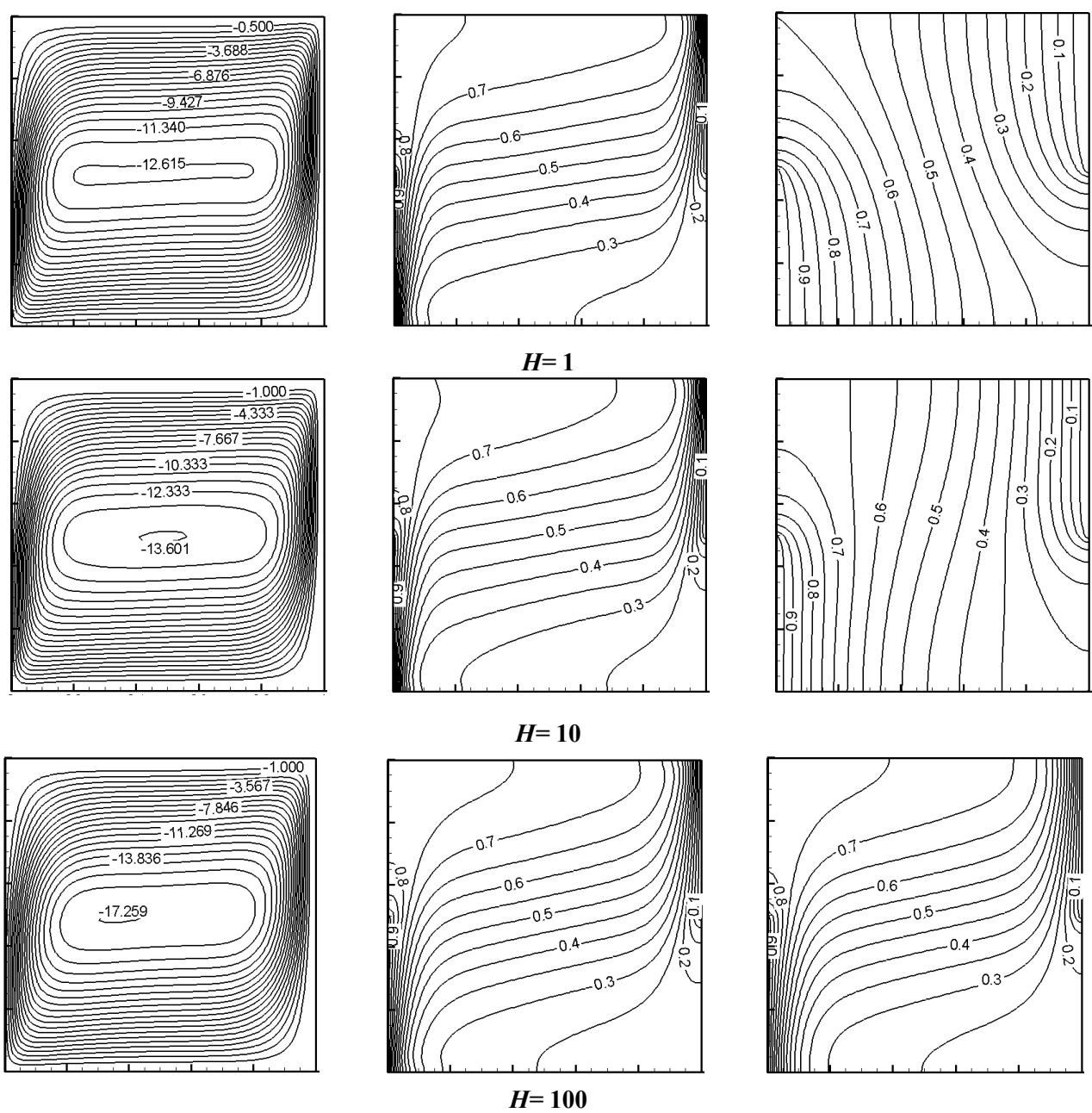

$$
D a=10^{-3}, R a=10^{6}, \gamma=1
$$

Fig. 2. Effect of $H$ on streamlines and isotherms for fluid (in the middle) and solid (on the right). Configuration:Bottom-Top.
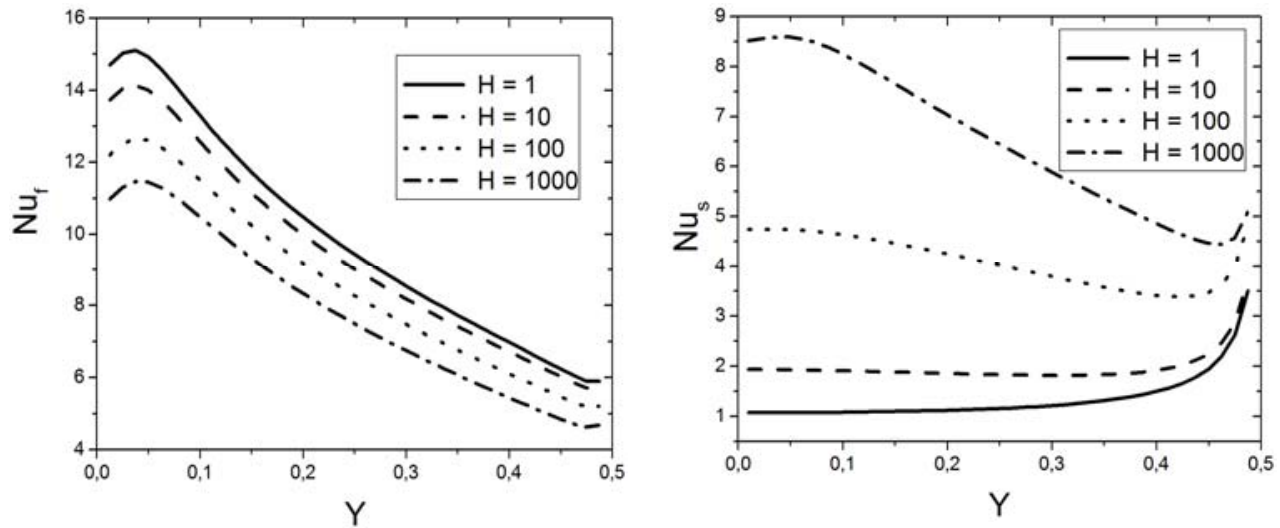

Fig. 3. Effect of $H$ on Nusselt number distributions for fluid $\left(N u_{f}\right)$ and solid $\left(N u_{s}\right)$, Configuration: Bottom-Top. 

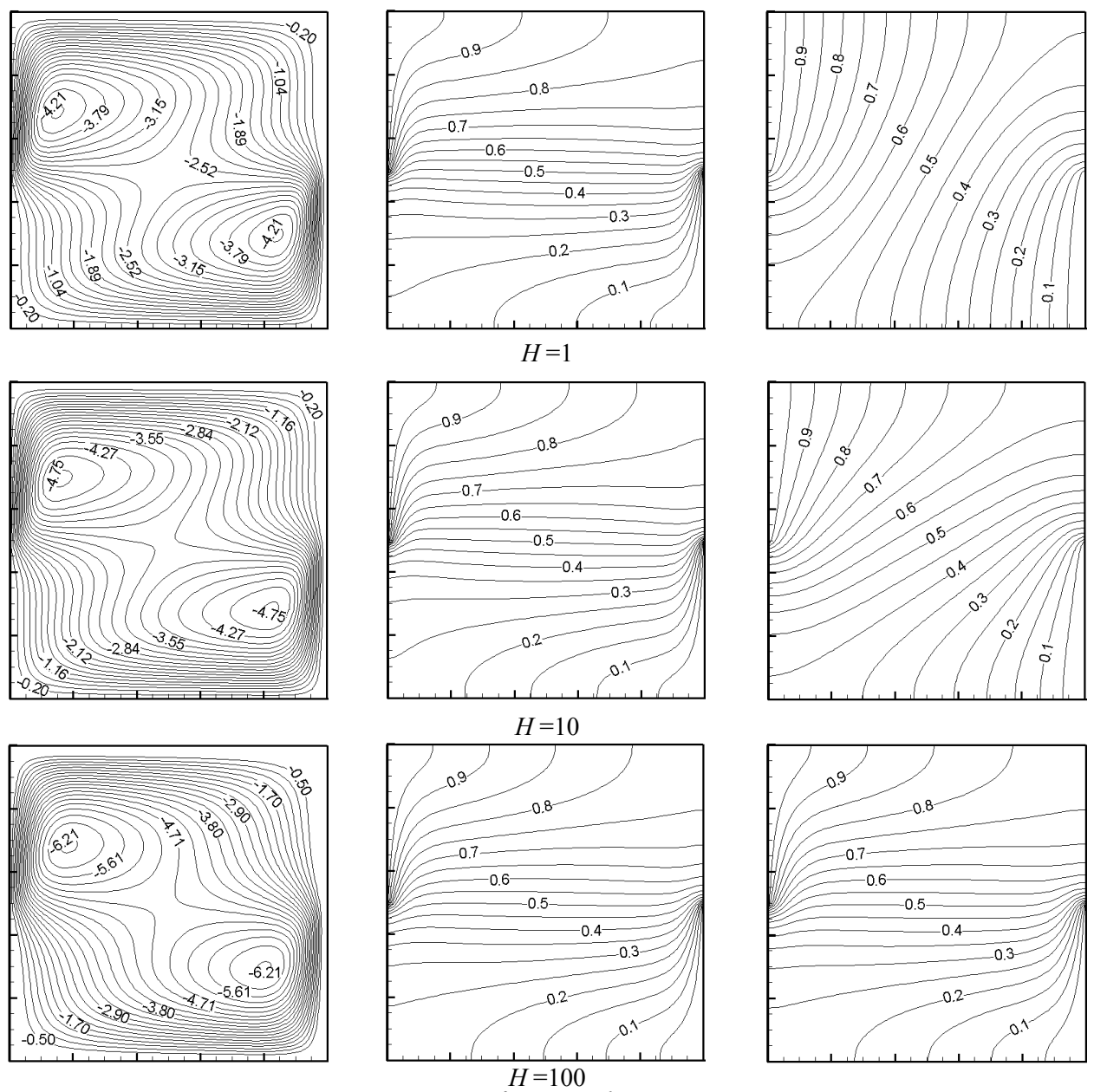

$\mathrm{Da}=10^{-3}, \mathrm{Ra}=10^{6}, \gamma=1$

Fig. 4. Effect of $H$ on streamlines and isotherms for fluid (in the middle) and solid (on the right). Configuration: Top-Bottom.
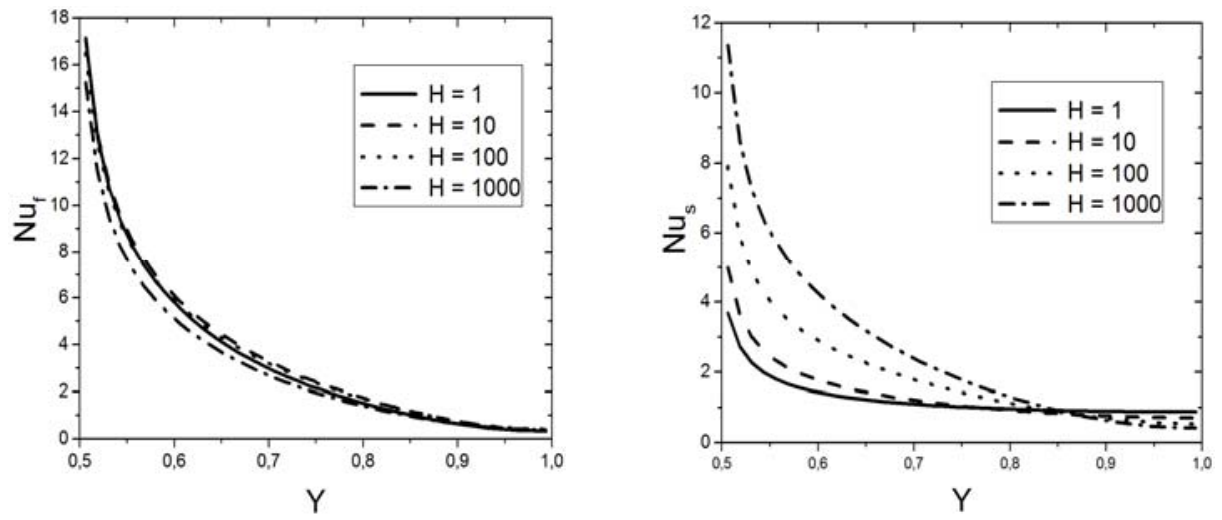

Fig. 5. Effect of $H$ on Nusselt numbers for fluid $\left(\mathrm{Nu}_{\mathrm{f}}\right)$ and solid $\left(\mathrm{Nu}_{\mathrm{s}}\right)$, Configuration: Top-Bottom.

Fig. 7 illustrates the effect of $H$ at different values of thermal conductivity ratio zon the average Nusselt numbers for fluid and solid as well as the maximum absolute value of the solid-to-fluid temperature difference inside the cavity for three thermally active locations in which $D a=10^{-3}$ and $R a$ $=10^{6}$. An overview of the figure reveals that the average Nusselt number for fluid decreases steadily with $H$ for low values of $\gamma$. In the contrary, the average Nusselt number for the solid phase increases with increasing values of $H$. For $\gamma \geq 10$, the average Nusselt number for the fluid phase becomes almost independent of $H$, while the average Nusselt number for the solid phase increases up to $H \leq 200$ and then it becomes independent of $H$. 

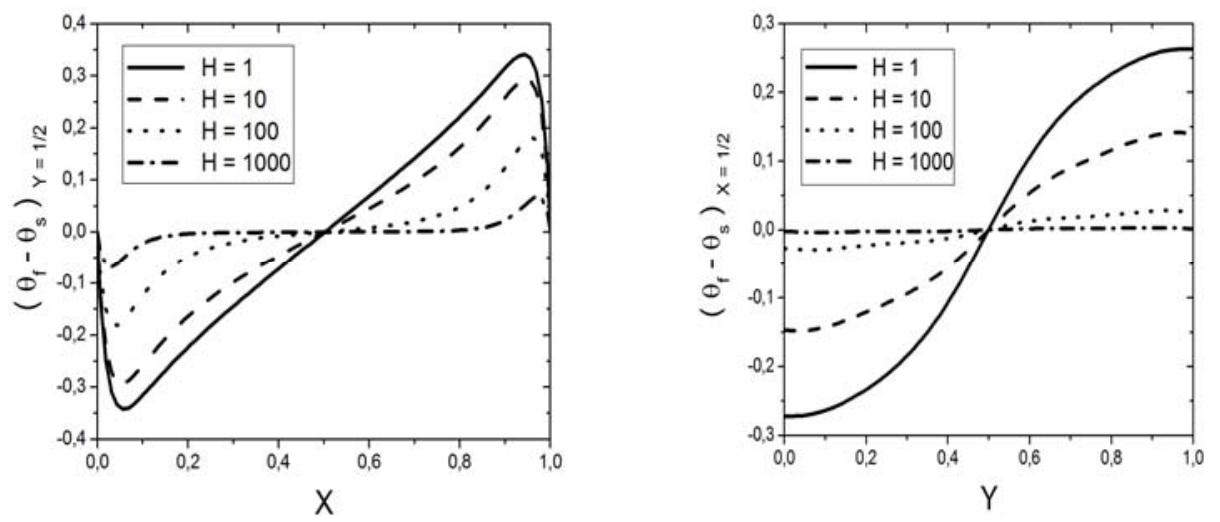

Top-Bottom heating
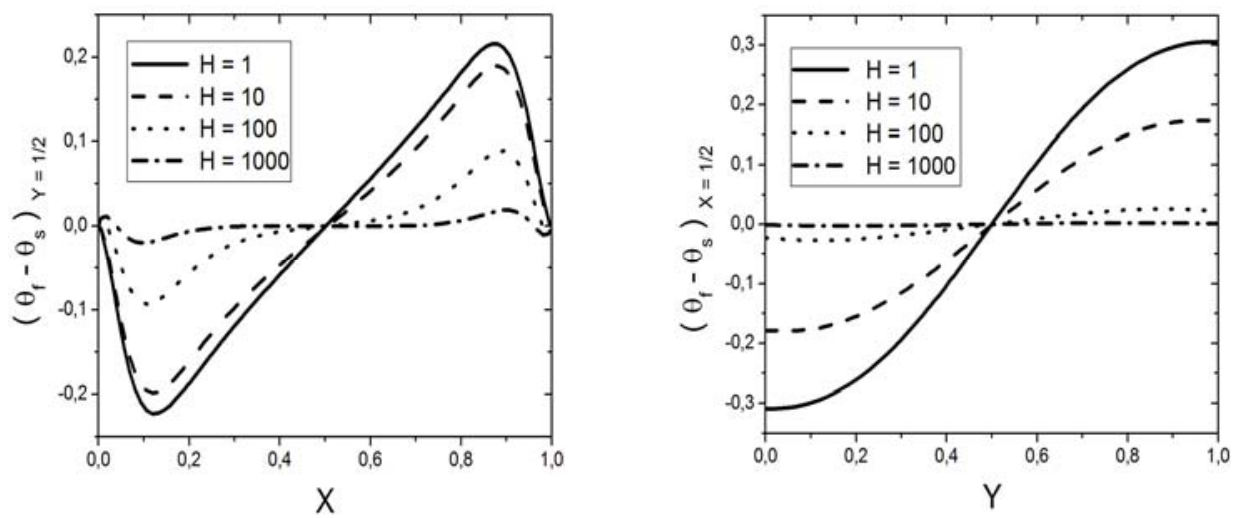

Bottom -Top heating
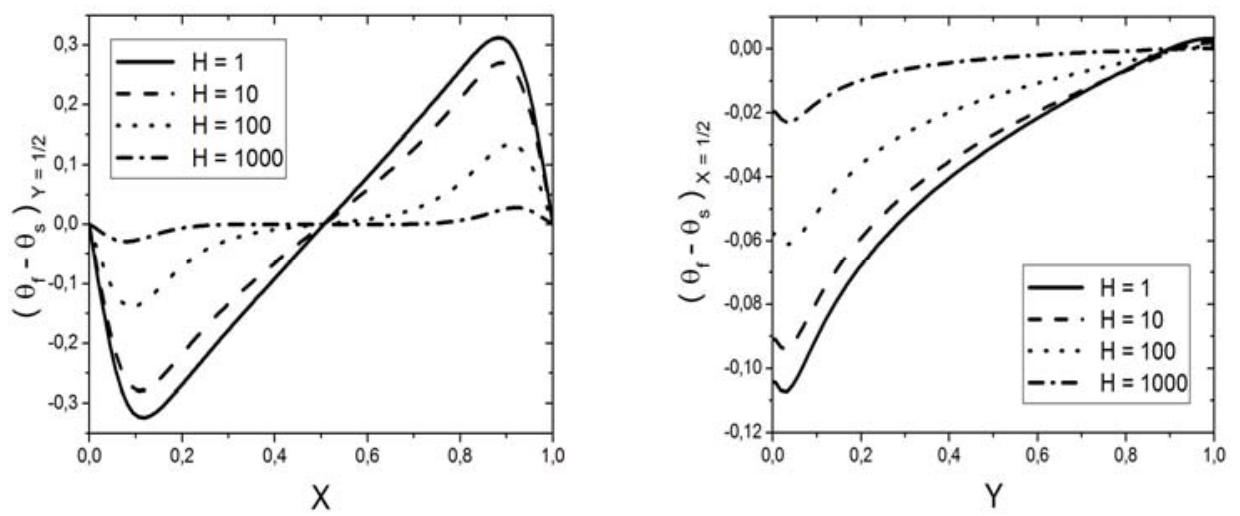

Total heating

Fig. 6. Solid-to-fluid temperature at the horizontal mid-plane ( $Y=0.5$ on the left) and the vertical midplane $\left(X=0.5\right.$ on the right) for different values of $H$ at $D a=10^{-3}, R a=10^{6}$ and $\gamma=1$.

On the other hand, the difference between average Nusselt number of fluid and solid phases decreases with increase of $\gamma$ and $H$ until both fluid and solid Nusselt numbers are equal. At this stage, the solid and fluid phases reach the thermal equilibrium state. Further, it is obvious that the heat transfer for the case with Bottom-Top heating and cooling produces a better heat rate compared to the case of TopBottom heating and cooling. Moreover, the heat transfer rate relating to Bottom-Top heating and cooling is comparable to the corresponding one produced by the total heating case. Finally, the corresponding maximum absolute value of the solid-to-fluid temperature difference inside the cavity confirms the above findings.

Fig. 8 shows the variations of average Nusselt number for fluid and solid with respect to Rayleigh number and the thermal conductivity ratio $\gamma$ for the two previous cases of thermally active locations and for the case with total heating and cooling. An overview of the figure reveals that the average Nusselt numbers for fluid and solid increase steadily with Rayleigh number. In particular, they 

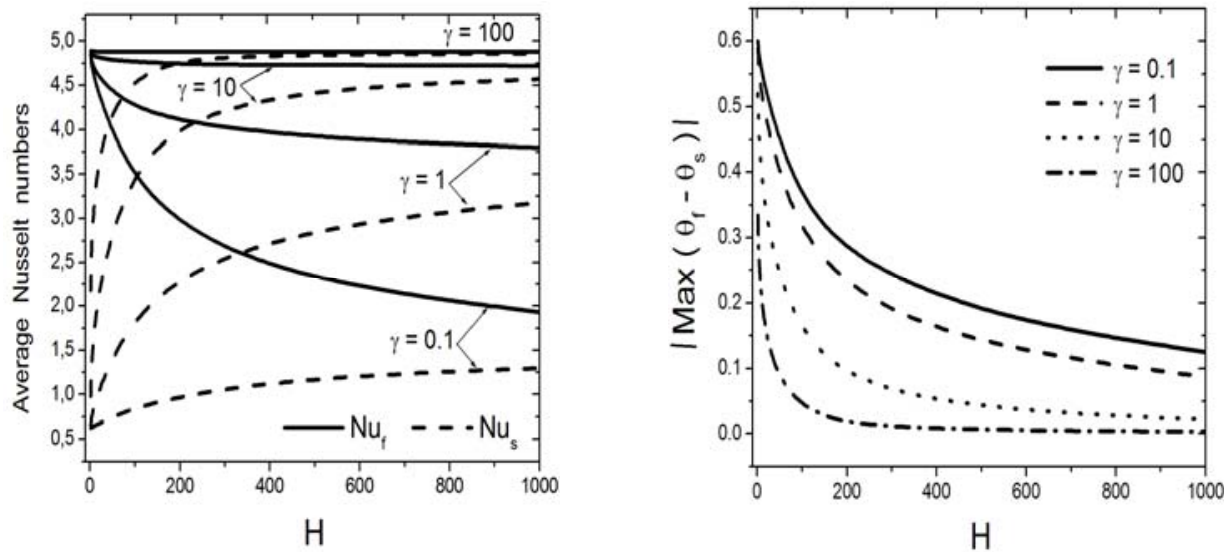

Bottom -Top heating
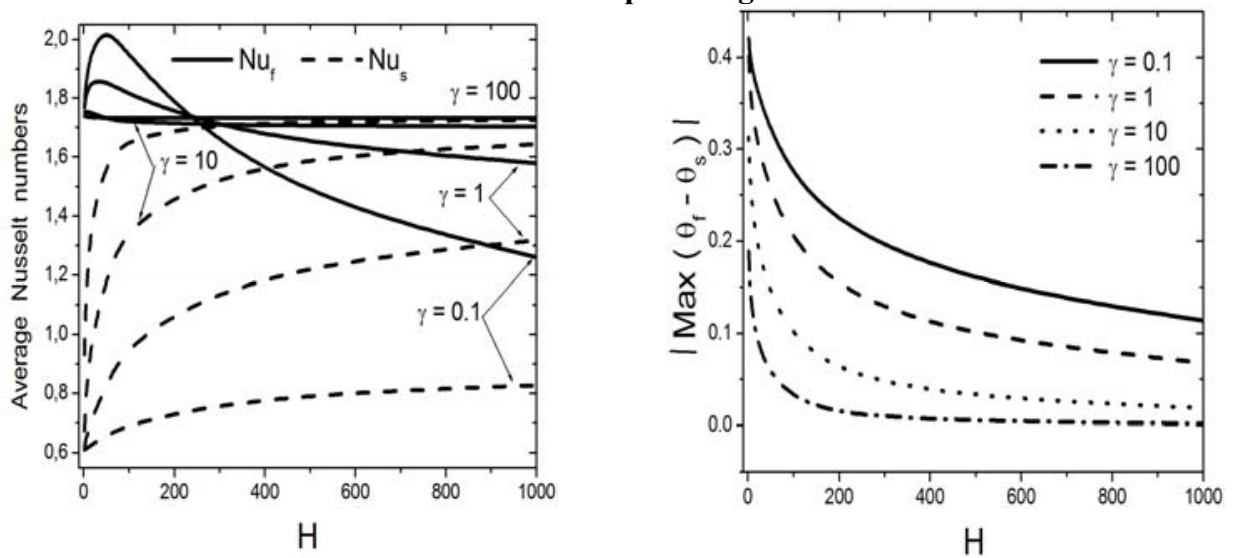

Top-Bottom heating
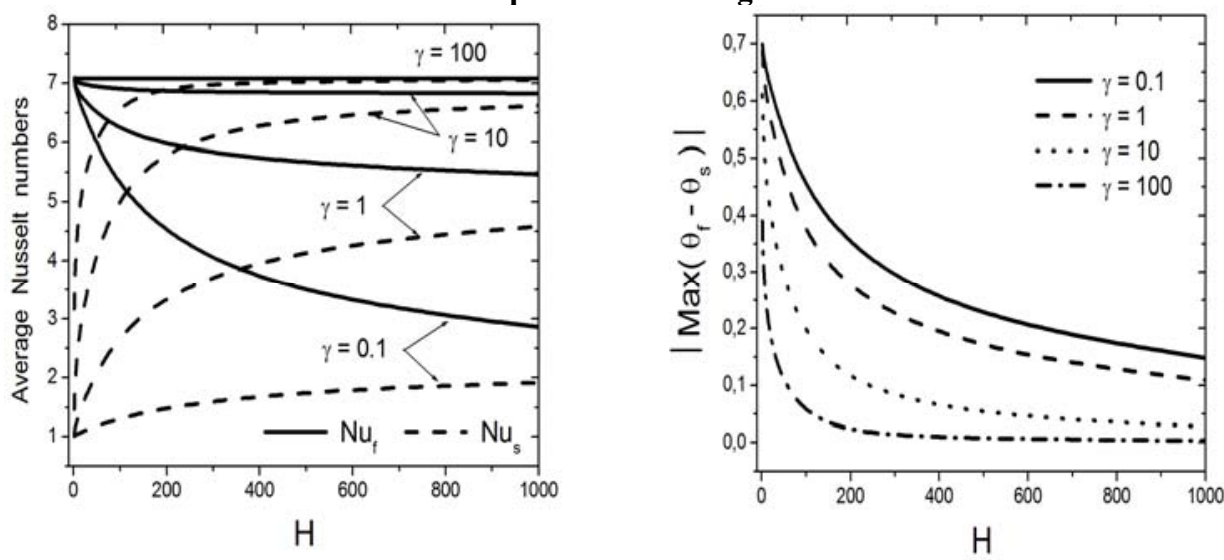

Total heating

Fig. 7. Average Nusselt number (on the left) and the maximum absolute value of the fluid-to-solid temperature difference (on the right) vs $H$ at $D a=10^{-3}, R a=10^{6}$ and $\gamma=1$.

increase steeply when $R a>10^{5}$, whereas when $R a<$ $10^{4}$ both Nusselt numbers maintain a constant and uniform value which correspond to prevailing conduction heat regime. In fact, for this case $(R a<$ $10^{4}$ ), the Nusselt number for fluid and solid are almost equal to 0.5 for partially heating and cooling and 1 for total heating and cooling. Also, the results clearly show that at low $\gamma$, the average Nusselt number for solid is constant and uniform irrespective of the Rayleigh number $(0.5$ for partial heating and cooling and 1 for total heating). The increase of thermal conductivity ratio $\gamma$ enhances heat exchange between solid and fluid phases, which can leads to thermal equilibrium state between the solid and fluid phases even though $H$ is low $(H=10)$ as one can see it for the case with $\gamma=100$. Again, it is clear that the minimum rate of heat transfer takes place when the arrangement of the heating-cooling is Top-Bottom, irrespective of $R a$ and $\gamma$, and that the local thermal equilibrium is 

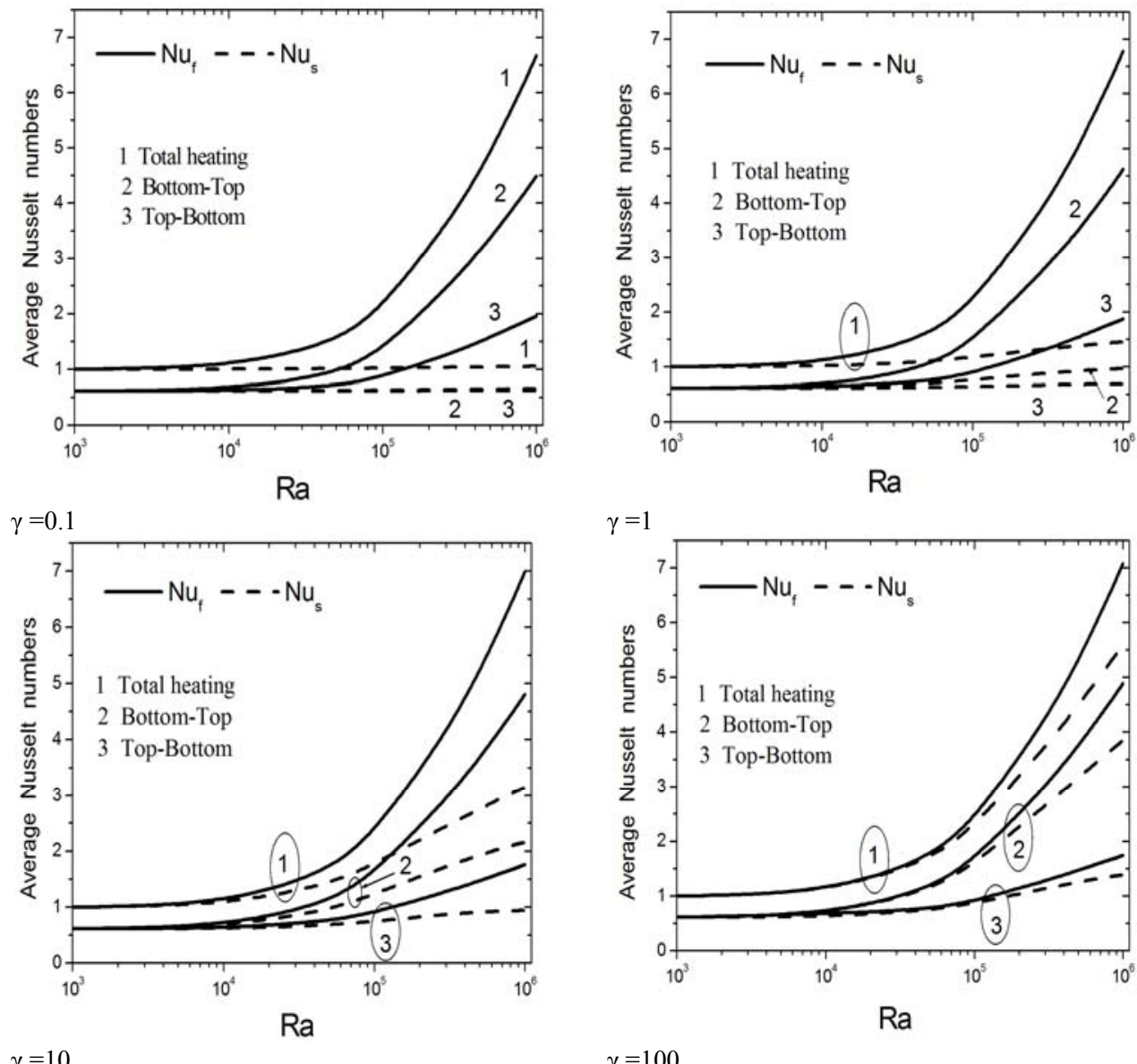

$\gamma=10$

Fig. 8. Average Nusselt number vsRa for different values of $\gamma$ at $D a=10^{-3}, H=10$.

the first achieved for a given value of $R a$ for this arrangement.

Fig. 9 illustrates the variation of average Nusselt number with respect to Darcy number. It can be seen that the fluid and solid average Nusselt numbers remain almost constant up to $D a=10^{-5}$ irrespective of $\gamma$ and the locations of the thermally active zone. Beyond this value, they reveal an increasing and then undergone a quasi-uniform variation for $D a \geq 10^{-3}$. It is interesting to observe that the difference between the fluid and solid Nusselt numbers in this case is higher than the corresponding one in Fig. 8 indicating that nonequilibrium state prevails over a wide range of Darcy number irrespective of $\gamma$ values. The effect of Forchheimer number on average Nusselt number is depicted in Fig. 10 for different values of thermal conductivity ratio $\gamma$. It can be observed from the figure that for the case with Top-Bottom heating and cooling, the Forchheimer number has no effect on the heat transfer rate of the solid phase and the liquid phase.. For the case with Bottom-Top and total heating and cooling, the effect of Forchheimer number on heat transfer rate is negligible. For this reason, the value of Forchheimer number has been fixed for all results obtained previously. Similar observation has been reported by Bera et al. (2014).

\section{Conclusion}

Numerical investigation using finite volume method is conducted to study the effect of partially heatingcooling zones on natural convection in a square porous cavity under the influence of local thermal non-equilibrium state (LTNE). The analysis is mainly focused on the location of the heatingcooling zones on the natural convection whitin the cavity as a function of control parameters, such as the inter-phase heat transfer coefficient, Darcy and Rayleigh numbers and the modified conductivity ratio. It was observed that:

1. The arrangements of active portions play an important role on the fluid flow and heat transfer rate.

2. The highest heat transfer rate was obtained for the Bottom-Top arrangement while the lowest heat transfer rate is obtained for the Top-Bottom arrangement.

3. Heat transfer rate increases with increasing Rayleigh and Darcy numbers irrespective of heating-cooling location zones.

4. For a given value of $H$ and at low values of $R a$ and $D a$ numbers, the local thermal equilibrium state between fluid and solid phases prevails whatever. 

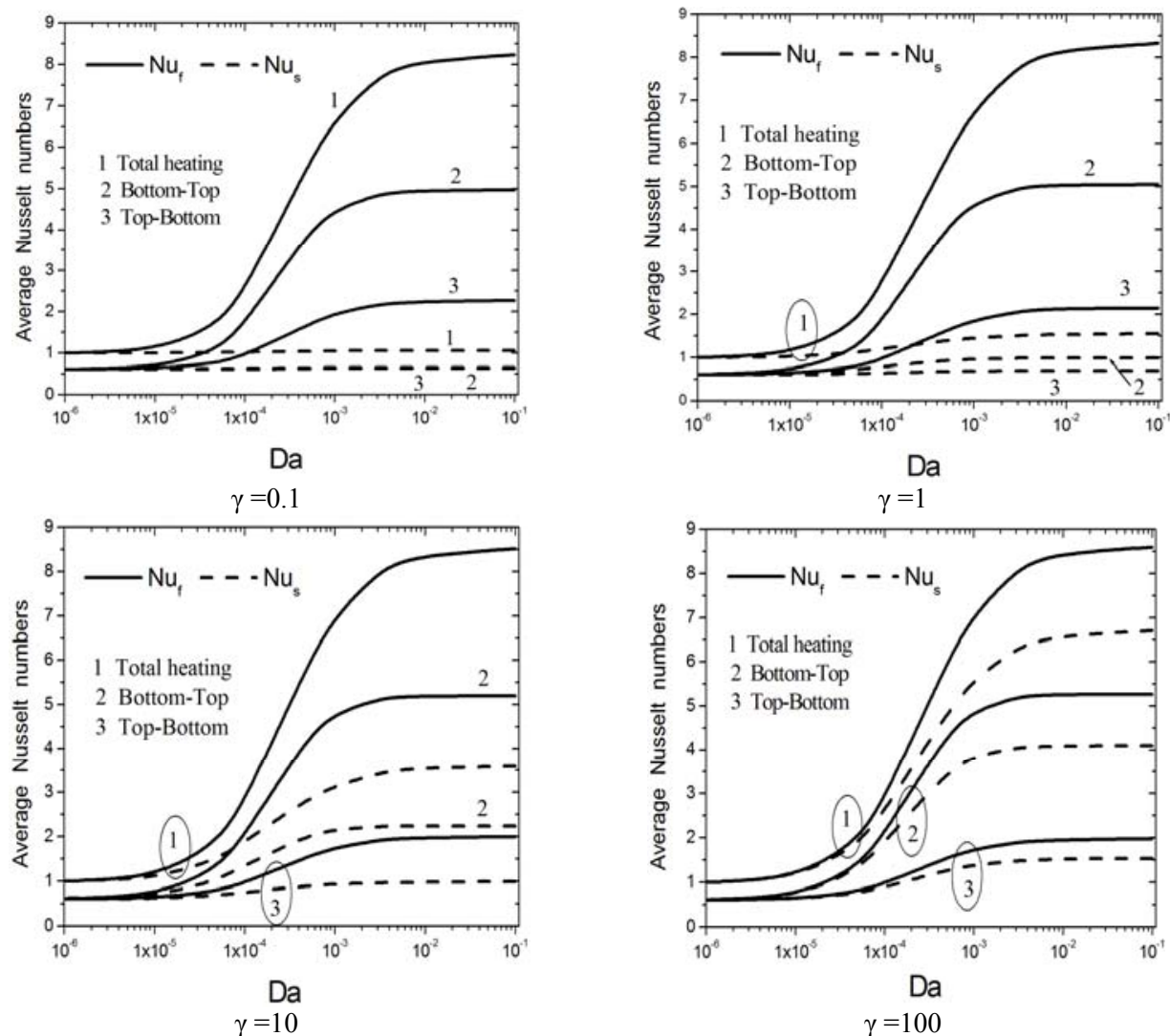

Fig. 9. Average Nusselt number vs $D a$ for different values of $\gamma$ at $R a=10^{6}, H=10$.
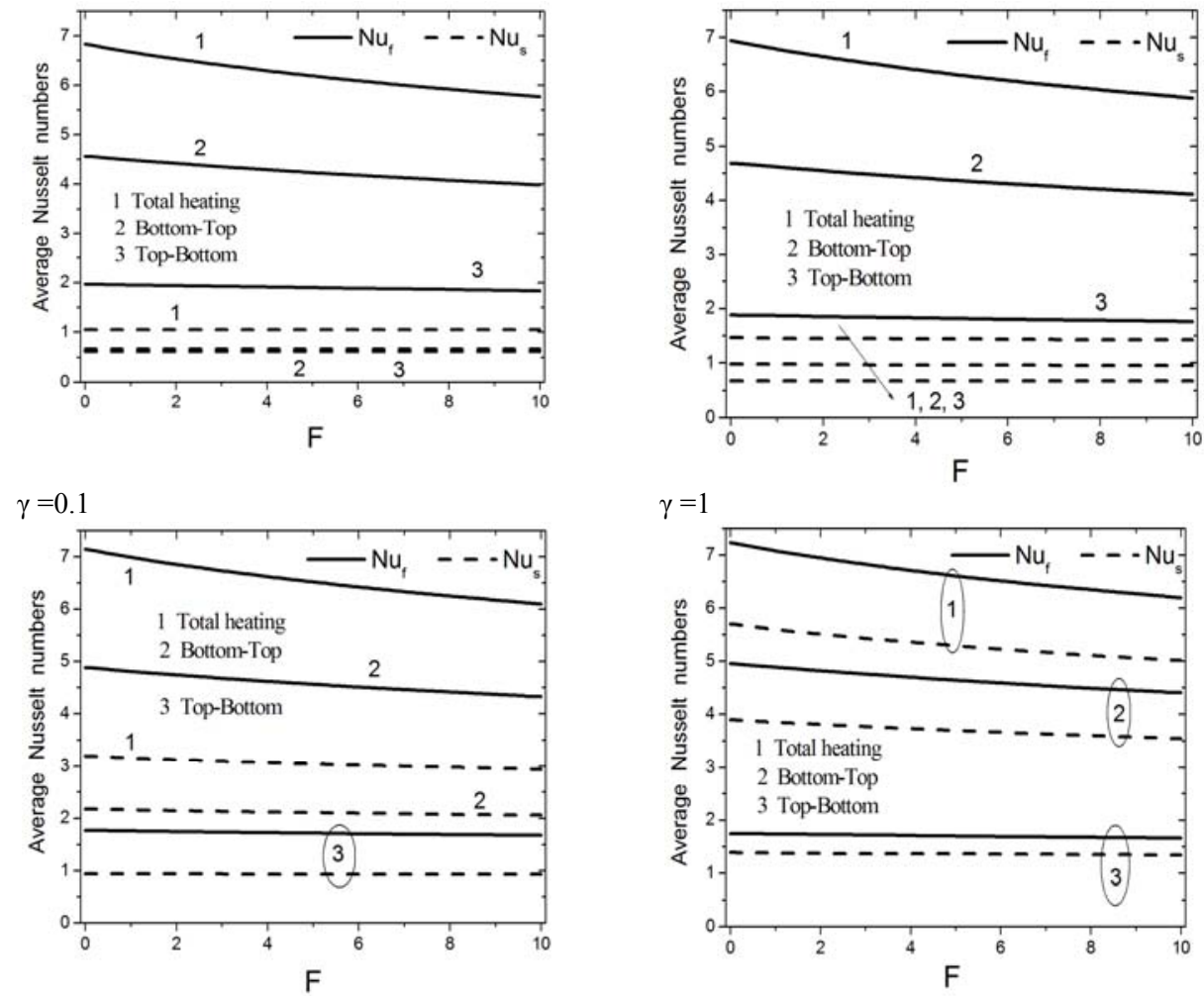

$\gamma=10$

$\gamma=100$

Fig. 10. Average Nusselt number vs Forchheimer number for different values of $\gamma$ at $R a=10^{6}, D a=10^{-3}$, $\boldsymbol{H}=\mathbf{1 0}$. 
the thermal conductivity ratio value $(\gamma)$. By increasing the $D a$ and $R a$ numbers the difference between Nusselt numbers increases and the rate of increases of this difference with respect to $D a$ is more important than the corresponding one for $R a$

5. As $H$ increases, the solid-to-fluid temperature difference decreases and approaches zero, indicating that the LTE state is verified anywhere in the cavity.

6 . In the case of total heating, the solid-to-fluid temperature difference (at $X=1$ ) decreases gradually from $Y=0$ to $Y=1$ where the solid-to-fluid temperature difference is almost zero for all values of $H$ contrary to the case with partially heatingcooling zones where the distribution of temperature difference varies symmetrically with respect to $Y$ axis.

\section{REFERENCES}

Abdedou, A. and K. Bouhadef (2015). Comparison between two local thermal non-equilibrium criteria in forced convection through a porous channel. Journal of Applied Fluid Mechanics, 8(3), 491- 498.

Badruddin, I. A., Z. A. Zainal, P. A. Aswatha Narayana and K. N. Seetharamu (2007).Numerical analysis of convection conduction and radiation using a nonequilibrium model in a square porous cavity. International Journal of Thermal Sciences 46, 20-29.

Baytas, A. C. (2003). Thermal non-equilibrium natural convection in a square enclosure filled with a heat-generating solid phase, non-Darcy porous medium. International Journal of Energy Research 27, 975-988.

Baytas, A. C. and I. Pop (2002). Free convection in a square porous cavity using a thermal nonequilibrium model. International Journal of Thermal Sciences 41, 861-870.

Berra, P., S. Pippal and A. K. Sharma (2014).A thermal non-equilibrium approach on double diffusive natural convection in a square porous-medium cavity. International Journal of Heat and Mass Transfer 78, 1080-1094.

Calmidi, V. V. and R. L. Mahajan (2000).Forced convection in high porosity foams. Transactions of the ASME Journal of Heat Transfer 122, 557-565.

Dincov, D. D., K. A. Parrott and K. A. Pericleous (2004).Heat and mass transfer in two-phase porous materials under intensive microwave heating. Journal of Food Engineering 65, 403412.

Ingham, D. B. and I. Pop (Eds.) (2002). Transport phenomena in porous media. Vol. 2.

Nield, D. A., and A. Bejan (1999).Convection in porous media. Springer, New York (Second ed).

Patankar, S. V. (1980). Numerical Heat Transfer and Flow. Hemisphere Publication Company, New York.

Pippal, S. and P. Bera (2013). A thermal nonequilibrium approach for $2 \mathrm{D}$ natural convection due to lateral heat flux: Square as well as slender enclosure. International Journal of Heat and Mass Transfer 56, 501515.

Saeid, N. H. (2004). Analysis of mixed convection in a vertical porous layer using a thermal nonequilibrium model. International Journal of Thermal Sciences 47,5619-5627.

Vafai, K. (Ed.) (2000). Handbook of Porous Media.Marcel Dekker, NewYork.

Virto, L., M. Carbonell, R. Castilla and P. J. Gamez-Montero (2009).Heating of saturated porous media in practice: Several causes of local thermal non-equilibrium. International Journal of Heat and Mass Transfer 52, 54125422 .

Wu, F., W. Zhou and X.Ma (2015). Natural convection in a porous rectangular enclosure with sinusoidal temperature distributions on both side walls using a thermal nonequilibrium model. International Journal of Heat and Mass Transfer 85, 756-771.

Zorlia, S. E., and A. C. Rubiolo (2005).Mathematical modeling for immersion chilling and freezing of foods. Part I: Model development. Journal of Food Engineering 66, 329-338. 